\section{A generalized polynomial model for perception of exponential series}

\author{
GREGORY V. JONES \\ University of Oxford, Oxford, England
}

Wagenaar and Timmers (1978) have recently reported an experiment investigating subjective extrapolation whose results may appear to be incompatible with the proposals of Jones (1977). However, the present article gives mathematical, physical, psychological, and statistical reasons for retaining the polynomial theory of Jones (1977). Levels of subjective extrapolation of exponential series can be as well described relative to the simplest form of curvilinear polynomial extrapolation, the quadratic, as by the exponential model of Wagenaar and Sagaria (1975). In addition, it is shown that extrapolation from other types of series may be considered in a similar manner.

In several articles concerning subjective extrapolation from sets of numbers which conform to simple exponential series, Wagenaar and his colleagues (e.g., Timmers \& Wagenaar, 1977; Wagenaar \& Sagaria, 1975; Wagenaar \& Timmers, 1978, in press) have consistently found that subjectively extrapolated values differ sizably from exponentially extrapolated ones. They attribute such deviations to subjective error: "Whenever subjects fail to correctly extrapolate a time series, one would like to know whether either one or both stages [of processing] contribute to the inaccuracy" (Wagenaar \& Timmers, 1978, p. 182, italics added). Mathematically, however, extrapolation cannot be said to be correct or incorrect. There are an infinity of possible extrapolations, and choice between them can only be made on the basis of a hypothesis concerning the process that generated the numbers. In these experiments, the instructions appear to have been intended to suggest an exponential hypothesis indirectly by mentioning, before the presentation of the numerical series, "that many processes in our environment, such as pollution, inflation, and energy consumption, show marked growth tendencies" (Wagenaar \& Timmers, 1978, p. 183). In practice, however, pollution, inflation, and energy consumption often do not demonstrate exponential growth. If an index of this type of process initially took the values $3,7,20,55$, and 148 in 5 successive years (as in one of the numerical series used), other forces would normally intervene to prevent unabated exponential growth to a value of

The author is grateful to an anonymous reviewer for helpful comments. Requests for reprints should be sent to Gregory V. Jones, University of Oxford, Department of Experimental Psychology, South Parks Road, Oxford OXI 3UD, England.
Table 1

U.K. Natural Gas Consumption with Exponential Fitted To First 5 Years

\begin{tabular}{ccc}
\hline Year & Consumption* & Exponential \\
\hline \multicolumn{3}{c}{ First 5 Years } \\
1967 & 2.1 & 2.3 \\
1968 & 4.7 & 4.4 \\
1969 & 9.2 & 8.5 \\
1970 & 17.6 & 16.4 \\
1971 & 28.4 & 31.5 \\
& Second 5 Years & \\
1972 & 40.3 & 60.5 \\
1973 & 43.6 & 116.3 \\
1974 & 52.1 & 223.5 \\
1975 & 54.5 & 429.3 \\
1976 & 57.9 & 824.7 \\
\hline
\end{tabular}

Note-Data are from the Central Statistical Office (1977, p. 72). *Each unit is equivalent to a million tons of coal.

22,026 in a further 5 years. As an example, Table 1 shows the inland U.K. consumption of natural gas over the 10 years 1967 to 1976 . It can be seen that over the first 5 years the data agree closely with the best-fitting exponential $y=1.2042 \exp (.65292 \mathrm{x}), \mathrm{x}=$ 1 to 5 , but that over the next 5 years this exponential grossly overpredicts actual consumption. Thus, for both mathematical and physical reasons, subjective extrapolation at less than exponential levels cannot be said to be "underestimated" (Wagenaar \& Timmers, 1978, p. 182).

The question of how, in fact, exponential series are subjectively extrapolated is a purely empirical one. Thus, the model proposed by Wagenaar and his colleagues (e.g., Wagenaar \& Timmers, 1978), in which the exponential growth factor $\exp (b)$ is said to be underestimated as $\alpha \exp (\beta b)$, has to be compared empirically on equal terms with other competing models. Jones (1977) proposed that subjective extrapolation was best described by a low-order polynomial, and specifically (for the initial five-point series used in the experiments of Wagenaar \& Sagaria, 1975) the quartic collocation polynomial. It was shown that this function fit experimental data reasonably well (a simpler best-fitting quadratic fit them even better), and had the substantial advantage that its application required the estimation of no parameter values from the experimental data, whereas that of Wagenaar and Sagaria required the estimation of two, $\alpha$ and $\beta$.

\section{Generalized Polynomial Model}

In spite of the success of the unelaborated polynomial hypothesis, it is clear that it lacks the generality to represent systematic differences to be expected among, for example, the extrapolations of different subjects. Similarly, Wagenaar and Timmers (1978) have shown that increasing the number of initial 
points in series which are said to be of equal duration leads to lower subjective extrapolations. For example, if the initial series $(3,20,148),(3,7,20,55,148)$, and $(3,5,10,20,39,76,148)$ are said to be of 5 years' duration each, then the average extrapolated values after another 5 years (i.e., for Year 10) are approximately 853,419 , and 283 , respectively. Generalizing the polynomial model to account for such findings, it is proposed that subjects' extrapolations from exponential series consist of proportional functions of quadratic extrapolations; that is, $S_{i}=k Q_{i}$, where $S_{i}$ is the subjective extrapolation and $Q_{i}$ is the least-squares quadratic extrapolation. The value of $\mathbf{k}$ is hypothesized to be independent of the rate of increase of the initial series, but to vary with its mode of presentation (e.g., whether it has many or few points, or is graphical rather than digital) and also over subjects.

The model was tested by applying it to the extrapolation of initial series consisting of $n=3,5$, or 7 equally spaced members of the four functions described by $y=\exp (b x)$, with $b=1.0,1.2,1.4$, and 1.6; the terms of the three series for $b=1.0$ were

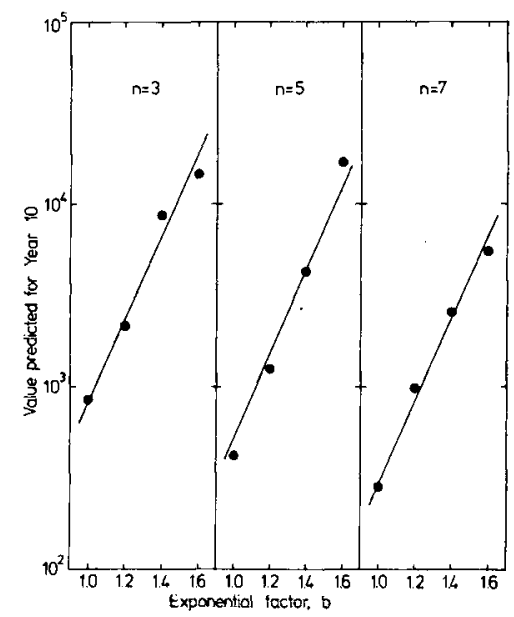

Figure 1. Subjective extrapolations (circles) compared with predictions of generalized polynomial theory (lines) for $n$ initial values conforming to exp(bx).

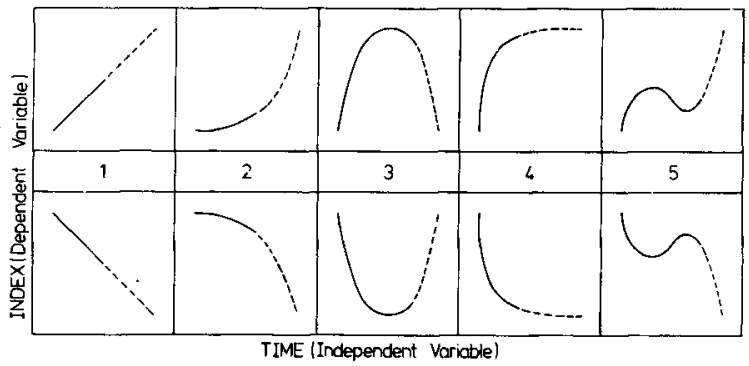

Figure 2. Polynomial extrapolation for the five categories of Table 2. Continuous and dashed lines represent initial series and extrapolations, respectively. Upper and lower panels show initially positive and negative gradients, respectively.
Table 2

Dependency of Polynomial Extrapolation on Gradient of Initial Series

\begin{tabular}{ccc}
\hline Category & Magnitude of Gradient & Extrapolation \\
\hline 1 & Constant & Linear \\
2 & Increases & Quadratic \\
3 & Decreases Until Sign Reverses & Quadratic \\
4 & Decreases But No Sign Reversal & Quadratic-Linear \\
5 & Sign Reverses Twice & Cubic \\
\hline
\end{tabular}

given earlier. If the generalized model correctly represents extrapolative processing, then $\mathrm{k}$ should be constant for different values of $b$, but should vary systematically with the value of $n$. Figure 1 shows the fit of the model to the data averaged over subjects reported by Wagenaar and Timmers (1978), with $k$ taking the values $.86, .55$, and .31 for $n=3,5$, and 7 respectively. It can be seen that the agreement between data and model is, indeed, very close.

The present model appears to be preferable to that proposed by Wagenaar and his colleagues for three reasons. First, it is more parsimonious in requiring that only one parameter, $k$, be estimated from the data instead of two. Second, it is plausible that subjects should process the initial series relative to the analytically simplest form of accelerative function, the quadratic, rather than relative to the exponential function, which, in contrast, can be regarded as a polynomial of an infinitely high degree since $\exp (\mathrm{x})$ $=1+x / 1 !+x^{2} / 2 !+\ldots+x^{m} / m !+\ldots$ Third, the polynomial model has the advantage that it may also be applied to extrapolation from a wide range of series which are distributed other than exponentially, as follows.

\section{Extrapolation from Different Types of Series}

According to the present model, extrapolations from exponential series are made relative to the bestfitting quadratic. It is parsimonious to hypothesize that the same type of extrapolation occurs for any other type of series which also constitutes an accelerative function. This hypothesis is expressed as Category 2 of Table 2 . In this table, the logic of the present model is extended systematically to several other basic categories of initial functions.

It is assumed that in each case a person extrapolates relative to the lowest-order polynomial which is compatible with the direction of any changes in the value of a function's gradient over the initial series. The resulting polynomial will hence be linear for Category 1 (initial straight line), quadratic for Category 2 (as earlier) and for Category 3 (maximum or minimum), and cubic for Category 5 (maximum and minimum, or point of inflection). The case in which the magnitude of the gradient of the initial series decreases, but does not change sign, is exceptional (Category 4). Here full quadratic extrapolation 
would require a person at some point to reverse the sign of the gradient. Instead, it is postulated that extrapolation is relative to the quadratic only until the magnitude of its gradient has decreased to zero; after that point, the gradient remains constant rather than being reversed in sign. The five categories are illustrated in Figure 2 for both initially rising and initially falling series.

At least one limitation of the model is apparent. This is that it is unlikely to hold when the initial series contains several maxima and minima, since such series normally suggest periodic functions rather than high-order polynomials. It remains to be investigated whether the model nevertheless successfully represents subjective extrapolation from other simpler series in addition to the exponential.

\section{REFERENCES}

Central Statistical Office. Economic trends: Annual supplement. London, England: Her Majesty's Stationery Office, 1977.

Jones, G. V. Polynomial perception of exponential growth. Perception \& Psychophysics, 1977, 21, 197-198.

TrMmers, H., \& WAGENAAR, W. A. Inverse statistics and misperception of exponential growth. Perception \& Psychophysics, $1977,21,558-562$.

Wagenaar, W. A., \& Sagaria, S. D. Misperception of exponential growth. Perception \& Psychophysics, 1975, 18, 422-426.

WAgenaAR, W. A., \& TIMmERs, H. Extrapolation of exponential time series is not enhanced by having more data points. Perception \& Psychophysics, 1978, 24, 182-184.

WagenaAR, W. A., \& Timmers, H. The pond- and duckweed problem: Three experiments on the misperception of exponential growth. Acta Psychologica, in press.

(Received for publication October 23, 1978; accepted November 15, 1978.) 